\title{
A FRAME OF REFERENCE FOR RESEARCH OF A BLOCKCHAIN- BASED SOLUTION TO CORPORATE GRC-MANAGEMENT
}

\author{
Le Chen ${ }^{1}$
}

DOI: https://doi.org/10.31410/ITEMA.2020.31

\begin{abstract}
This society has faced many sorts of global challenges, especially the world of business and technological innovation, there's no greater aroused general argument nowadays than digital transformation (DT). Among production-oriented Corporates which many of them have begun to integrate digital technology into most areas of their business. How to well fulfill Corporate Governance, Risk and Compliance (GRC) while expanding existing businesses in the dramatic growth in DT environment has become a major challenge for all Corporates. Through a review of previous studying works and based on existing gaps the author evaluated whether there could be the feasibility of a blockchain-based technology being integrated as a solution to Corporate GRC-Management together with the theory of entire personnel's GRC responsibility system which would be parts of an agenda for the future research on this field and also contribute to Corporates in an overall strategic height level to embrace the coming of DT.
\end{abstract}

Keywords: Digital transformation, Corporate GRC-management, Software, Blockchain.

\section{INTRODUCTION}

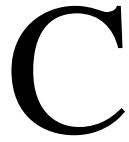

urrently, many Corporates desire to fully promote DT from top to bottom to develop a new business model to meet global changing and market requirements, however even now the existence of the assortment of GRC software products, how much actual value do they have for Corporate GRC-Management?

PricewaterhouseCoopers mentioned firstly the term "GRC" in 2004, then its diffusion was confirmed in the report which they stated, "The acronym "GRC" (governance, risk and compliance) has rapidly penetrated the business community over the last years. " (Racz et al., 2010 , p. 106). Herein in our paper we referred to the integrated approaches for Corporate GRCManagement and develop our work thanks to the study contributed by Racz and his research team, according to them (2010), "The final definition is as follows: " GRC is an integrated, holistic approach to organization-wide governance, risk and compliance ensuring that an organization acts ethically correct and in accordance with its risk appetite, internal policies and external regulations through the alignment of strategy, processes, technology and people, thereby improving efficiency and effectiveness. " (p. 112-113). This work was carried out in order to propose an innovative solution that supports Corporates fulfill the GRC-Management so as to maximize the safety of organizational operations with an integrated real-time concept through implement GRC in every process in all the internal organization to ensure all operations be carried out ethically, correctly, efficiently and effectively.

University for Foreigners "Dante Alighieri" of Reggio Calabria, Via del Torrione 95, Reggio Calabria, Italy 


\section{Objectives and research questions}

Although the studies of Nicolas Racz, Edgar Weippl, Andreas Seufert focused on analyzing "comparison with existing GRC software", but they still stated that, "The products' technology architectures mainly differ in their degree of integration, which is a key topic in future developments. Due to the lack of congruence, industry perspectives and existing state-of-theart GRC software should only very cautiously be applied in research." (Racz, N., Weippl, E., \& Seufert, A. (2011, January, pp. 1-10). This also confirmed that there is still no empirical research and hypotheses related to perspectives of application of the blockchain technology into Corporate GRC-Management. To fill this gap, specifically, we should take into account the following questions:

- What is blockchain technology actually good for?

- Why could blockchain technology be a solution for Corporate GRC-Management?

- How could a blockchain-based solution for Corporate GRC-Management well work?

\section{Methodology}

Three steps involved in conducting this study include Literature Review, Evaluation and Construction. The method we use in the study is qualitative, that is "Deductive ArgumentationNegative Requirements". First of all, let us set up a proposition: If the hypothesis "A is positive" holds, such that it makes the B come into existence. Similarly, if we let the hypothesis "A is negative" holds, hence it does not to make the existence of B. Namely,

$$
\text { If } A+, \text { then } B \text {; if } A-\text {, then not } B
$$

In detail, the premise assumption is that if the existing GRC software is perfect (if A+), then Corporates will no longer face any GRC-Management problems (then B). Based on this, similarly, we use the substitution method with negative hypothesis what means that we can substitute the negative hypothesis into the question. If no conclusion can be obtained, this negative hypothesis assumed must be necessary; if the conclusion can still be drawn, this negative hypothesis supposed is not necessary. That is to say, if we can prove the existing GRC software used by Corporates is not perfect (that is if A-), then for Corporates there are still GRC-Management problems to face (then not B). Therefore, we need to seek a better solution.

\section{What is blockchain technology actually good for?}

What is blockchain? Primavera De Filippi in his research stated, "A blockchain is a decentralized ledger (or state machine) that relies on cryptographic algorithms and economic incentives in order to ensure the integrity and legitimacy of every transaction (or state change). A copy of the blockchain is shared amongst all nodes connected to the network..." (Primavera De Filippi, 2016, p. 6). Nowadays, blockchain technology application exploration has extended to various fields after the application of pioneer Bitcoin:

- In the Internet of Things and Logistics field: This field is considered to be a promising application direction of blockchain (DHL Trend Research and Accenture, 2008).

- In the financial field: Such as Visa B2B Connect (Ye Chun Qing, 2016), Citibank's testing cryptocurrency dubbed "CitiCoin" (Biggs, J., 2015) has brought a revolutionary change. 
- In the insurance market: A typical application case is LenderBot ${ }^{2}$, a registered and customized micro-insurance product launched in 2016 by blockchain technology startup Stratumn, Deloitte and next-generation insurance platform LemonWay.

- In the Public services field (Akins et al., 2013): Blockchain is closely related to people's production and life in the fields of public management, energy, transportation, etc.

\section{Why could blockchain technology be a solution for Corporate GRC-Management?}

If blockchain technology has played an important role in promoting these fields, then we have reason to believe that it would also probably be significantly influential in the existing GRC software. Here we will verify the proposition of A- in the Expression (1) (see Section 2), that is, the imperfection of existing GRC software.

a. First of all, in practice, Corporates usually mix these three terms for using still without clear boundaries. Since there is still no "Authoritative Definition" by a World class Authority as OECD, there still exist different arguments about it among the scientific community on GRC, so, the basis of conceptual theory is inconsistent, brings more difficulty to subsequent development of GRC software. While the first peer-reviewed academic paper on the topic was published in 2007 by $\mathrm{OCEG}^{3}$ founder Scott L.Mitchell in the International Journal of Disclosure and Governance. This groundbreaking paper influenced an entire industry of software and services. According to Nicolas Racz, Edgar Weippl and Andreas Seufert (2010), "However to this day the concept behind the acronym has neither been adequately researched, nor is there a common understanding among professionals." (p. 106). And let us see, in the light of the extensive literature and available on the current scenario of GRC studied by Nicolas Racz, Edgar Weippl and Andreas Seufert (2010), "One in three of the analyzed publications offers a GRC definition (21\%). Two thirds of these definitions explain what is understood by GRC as an integrated concept (67\%). The remaining third disregards that the total might be more than the sum of its parts and confines itself to defining the three terms of governance, risk management and compliance separately. (12\%)" (p. 110).

b. Secondly, even under the premise of acknowledging the concept of integrated GRC, there are still problems in terms of development technology perspective. Because no releases of both an official concept and statistics on existing GRC software as indications by authoritative Organization (e.g. OECD). According to our preliminary statistics on the Internet, there exist no less than one hundred kinds various types of GRC software on the market, and each product is also very different, hence this requires a mainstream GRC software product with distinctive features to assist Corporates to fulfill GRC-Management.

c. Finally, GRC software has inherent defects as software that cannot be perfect. They stated, " $46 \%$ of the organizations in our survey have deployed GRC software that covers multiple governance, risk and compliance aspects. Only 29\% state that all GRC activities are consolidated in a single software platform, however." (Racz, N., Panitz, J., Amberg, M., Weippl, E., \& Seufert, A., 2010, p. 4). Even GRC software has been used into many organizations, we also worry about the difference from each other and their defects which are

LenderBot is a proof-of-concept micro-insurance platform that uses the bitcoin blockchain for insuring all sorts of things.

Founded in 2002, OCEG is headquartered in Phoenix, AZ. It's a non-profit think tank that is dedicated to achieving a world where every organization and every person strives to achieve objectives, address uncertainty and act with integrity. This approach to business, and to life, is what they call Principled Performance. 
the inherent components of software products. According to Humphrey ${ }^{4}$ (1999), "One of the things that really bothers me is the common software practice of referring to software defects by the term "bugs." In my view, they should be called "defects." " (p. 1). Anyhow, according to all the above discussion we have proved that the premise assumption is negative what is that if the existing GRC software is not perfect (that is if A-), consequently Corporates should still take into account the GRC-Management problems (then not B).

\section{How could a blockchain-based solution for Corporate GRC-Management well work?}

a. We precisely based on its characteristics that are Decentralization, Persistency, Anonymity and Auditability to proceed our study (Zheng, Z. et al., 2018, p. 357):

i. Decentralization. In conventional centralized transaction systems, the central trusted agency (e.g., the central bank) validated each transaction which generates the cost and the performance bottlenecks at the central servers (Zheng, Z. et al., 2018, p. 357). This is one of the highlights of our proposing to apply blockchain technology to solve GRCManagement which is particularly important in Corporate GRC-Management. It needs to be deployed and promoted by the highest decision-maker of the Corporate to make it a consensus for the development of the Corporate. Corporate GRC-Management which have been given the crucial functions into processes of every department within an organization is a shared responsibility of the entire Corporate, not simply performed only by the GRC-Management Department itself but all departments of the Corporate (will be elaborated further in following Point $b$ ).

ii. Persistency. "Since each of the transactions spreading across the network needs to be confirmed and recorded in blocks distributed in the whole network, it is nearly impossible to tamper. Additionally, each broadcasted block would be validated by other nodes and transactions would be checked. So any falsification could be detected easily." Stated by them (Zheng, Z. et al., 2018, p. 357). The case of Enron scandal became a typical case of Corporate Governance Failure. In the study of Borgia was stated "It is important because good or bad results are very much dependent on the way governance systems operate. It is topical because recent scandals have proved that today's ostensibly 'state-of-the-art' mode of governance is indeed inadequate. " (Borgia, F., 2005, p. 6).

iii. Anonymity. With a generated address each user can interact with the blockchain network. Further, many addresses could be generated by user in order to avoid expose the identity...So a certain amount of privacy on the transactions included in the blockchain is preserved thanks to this mechanism (Zheng, Z. et al., 2018, p. 357). Moreover, according to Racz, N., Panitz, J., Amberg, M., Weippl, E., \& Seufert, A. (2010), "Number, complexity and importance of GRC requirements steadily increase, resulting in companies undertaking various efforts to better face risks and to ensure the adherence to laws, regulatory standards and voluntarily imposed obligations (Menzies2006)." (p. 1), wherefore the security technology as digital signatures, identity authentication could be used for department supervision by its personnel (as Orphan blocks) so that the Corporate privacy could be well protected.

iv. Auditability. Since on the blockchain each of the transactions is validated and a timestamp is given. So the previous records through accessing any node in the distributed network can be easily verified and traced by users. The traceability and the transparency of the data stored in the blockchain is highly improved (Zheng, Z. et al., 2018, p. 357). It

Watts S. Humphrey (July 4, 1927 - October 28, 2010) was an American pioneer in software engineering, who was called the "father of software quality." 
establishes an open and healthy operating environment in which all departments work together in the method of sustainable development and transparency so as to optimize business benefits to the greatest extent.

b. In addition, herein we will elaborate a theory on how to further optimize Corporate GRCManagement. In 2018, a research report released by Accenture showed that despite the industry differences, in the digital transformation, leading companies in each industry stand out. Among them, the production-oriented enterprises in the automotive and parts and consumer electronics industries have the highest proportion of "digital transformation leaders". In the rapidly changing digital market environment, how to improve Corporate GRC-Management for existing businesses has become a great challenge.

i. As one component of GRC, the main risks confronted by Corporates, as we all know, can be divided into two categories: external risks and internal risks. External risks mainly include Political risks, Legal risks, Social and Cultural risks, Technological risks, Natural environmental risks, Market risks, Industrial risks, etc. Internal risks mainly include: Strategic risks, Management risks, Operational risks, technical risk, Financial risks, etc. To facilitate the following elaboration, here we will collectively call the various risks faced by the enterprise the Corporate Total Risk (abbreviated as "CTR"). Concerning production-oriented Corporates, generally, the organisational structure is mainly composed of Decision-making Organisation (Board), Procurement, Supply, Production, Logistics, Warehouse, Sales, Marketing, IT, Media, Accounting\&Finance, Administration, HR, Auditing, Law, R \& D, GRC and others with different functions. If the risk is distributed to various departments of an organisation, then let us see an example of Corporate's organisational structure shown on the right of Fig.1 below. Since we believe that the consistency of the structure between the blockchain and the Corporates is precisely same, and the comparison is as shown on Fig.1. As blocks are storage units one by one, which records all communication information of each block node within a certain period, just as both the communication and the cooperation between various departments of the Corporate.

Figure 1. Blockchain and Risk of organizational structure of production-oriented Corporate
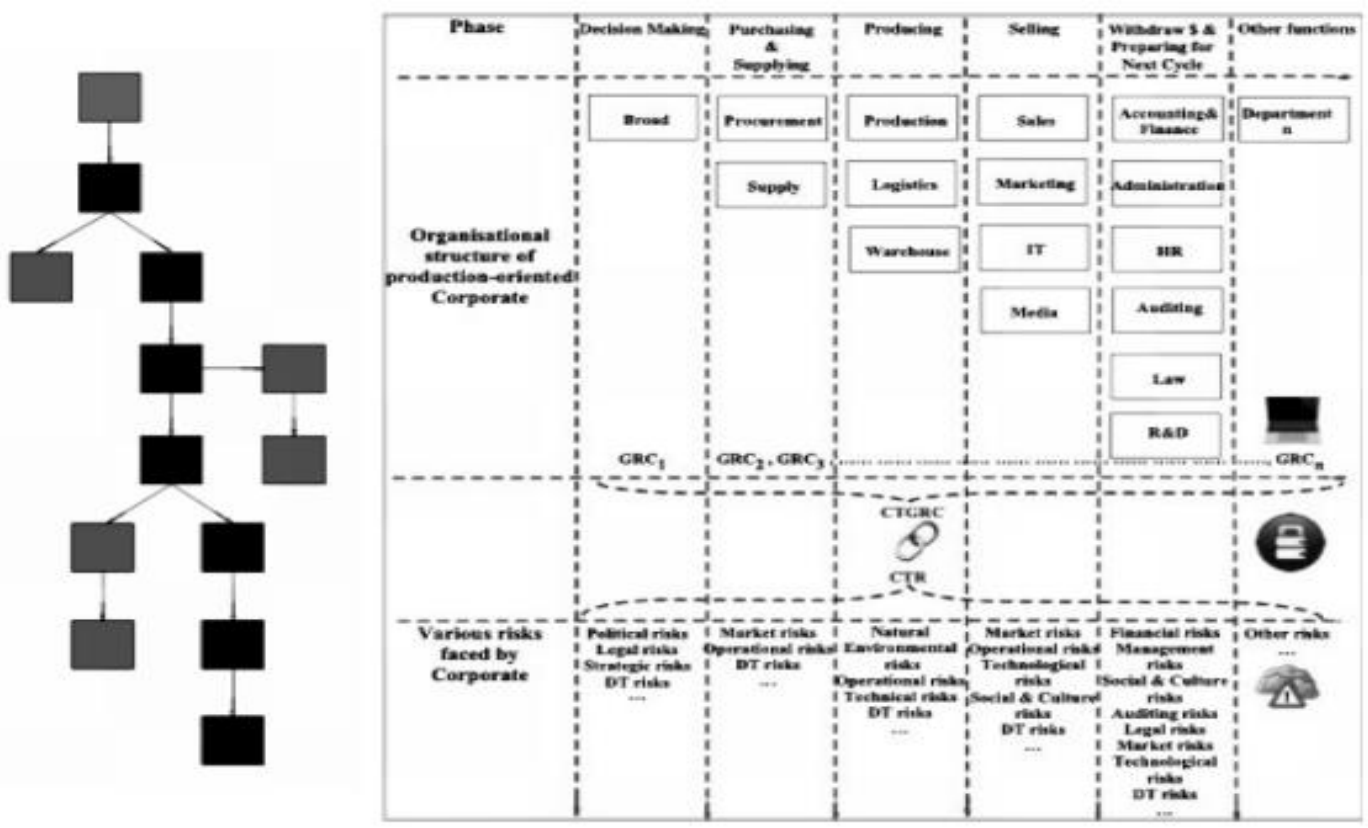
ii. Furthermore, we consider single department of a Corporate as every single chain. More vividly as shown on the left of above Fig.1 the Genesis block (the topmost one) to the current block is as Board of Corporates, the Main chain (start from 1 block below the topmost block, plus other 6 blocks running through the whole chain) is as the Heads of departments, and Orphan blocks (1 block branched on the left, and two pairs branched from the main chain located on its both outsides of the main chain) are as other Personnel. That is, if the probability of defects in one block or several blocks of Main chain is more higher, the security of the entire blockchain will be greatly reduced; just like a Corporate as shown on the above Fig.1, if the probability of the unfulfilled GRC (which is Corporate Total Risk (abbreviated as "CTR") in one or more departments is higher, then the higher the probability of unfulfilling Corporate Total GRC (abbreviated as "CTGRC"). Therefore, we believe that the core task for Corporate GRC-Management also the future research direction is to maximize the GRC of each department of the Corporate through blockchain technology. So to discover the form of the relationship between CTGRC and GRC of single department of the Corporate we can write it as:

$$
C T G R C=\sum_{i=m}^{n} G R C i=G R C 1+G R C 2+G R C 3+\ldots \ldots+G R C n
$$

Where $\mathrm{i}$ indicates the number of departments of the Corporate and $\mathrm{m} \geq 1$, and CTGRC is a constant $^{5}$. Generally, if the relationship between two variables $x$ and $y$ can be expressed in the form of $\mathrm{y}=\mathrm{k} / \mathrm{x}$ ( $\mathrm{k}$ is a constant, $\mathrm{k} \neq 0$ ), then $\mathrm{y}$ is called the inverse proportional function of $\mathrm{x}$. And as there exists the negative correlation between GTGRC and CTR, assume they are two variables and substitute them respectively into $\mathrm{Y}$ and $\mathrm{X}$, then the relationship of them can be expressed in the form as:

$$
\text { CTGRC }=k / C T R
$$

Where $\mathrm{k}$ is a constant, $\mathrm{k} \neq 0$, and CTR is a constant ${ }^{6}$. Consequently, we obtain the relationship between CTR and CTGRC which can be written as:

$$
\lim _{i=\infty}(C T G R C)=\lim _{i=\infty}\left(\frac{k}{C T R}\right)=k * \lim _{i \rightarrow \infty}\left(\frac{1}{C T R}\right)=0 \approx \operatorname{Min}[\text { CTR }]=\operatorname{Max}[\operatorname{CTGRC}]
$$

Obviously, substitute the Expression (3) into the Expression (4), when i the number of departments of the Corporate gets closer to infinity, namely, the responsibility of Corporate GRC is distributed into every department till each operation, then CTGRC gets closer to infinity, thus, the limit of the reciprocal of CTGRC converges to zero, then realize the minimization of CTR, namely, the maximization of CTGRC is fulfilled.

\section{Results}

Through a series of arguments above (Sections 3 to 5), we have demonstrated that the existing GRC software is not perfect, so Corporates still need to solve the GRC-Management problems. Therefore, Corporates should also take GRC-Management strategies into account to adapt to the new digital reality with new technologies to optimize Corporate GRC-management. According to the Expressions (2) and (4) we should consider the fact that is only by combining

\footnotetext{
5 CTGRC can be quantified by KPI and other performance evaluation indicators, so here we can regard it as a constant in a certain sense.

6 CTR can be quantified by the Risk Quantification model and other methods to measure risk, so here we can regard it as a constant in a certain sense.
} 
every department's GRC from every dimensions the Corporate should be able to in the true sense realize Corporate GRC-Management through an all-round cooperation. We stated a possible solution for existing GRC-Management based on both blockchain technical characteristics and the theory of entire personnel's GRC responsibility system.

\section{FUTURE RESEARCH DIRECTIONS}

There are still many practical aspects to continue to study:

- Subject to current laws. The supporting of laws and regulations, standards, supervision and management system should be strengthened, and especially we need to unify the understanding of integrated Corporate GRC-Management concept.

- At the technical level. Although it has the characteristics of greatly improving security, the application of Corporate GRC-Management still has the problem of embedding various processes in the GRC software to be solved, especially we need the prudence in the process of test. We also need to take into account in consideration of Whether these new technologies could own other potential technical advantages.

\section{CONCLUSION}

Overall, we hope that this study work to promote the official release of an integrated GRC definition from the authority level (e.g.OECD) as it has a magnificent meaning on the development of Corporate GRC-Management GRC; and to further elaborate our proposed framework in order to develop a solution based on blockchain technology and the theory of entire personnel's GRC responsibility system, especially when DT has emerged as one of the most important considerations in Corporate's Strategy-Making.

\section{ACKNOWLEDGEMENT}

The author is grateful to Prof. Mavilia Roberto and Prof. Loprevite Salvatore, for their valuable guidance.

\section{REFERENCES}

Akins, B. W., Chapman, J. L., \& Gordon, J. M. (2014). A whole new world: Income tax considerations of the Bitcoin economy. Pitt. Tax Rev., 12, 25. https://doi.org/10.5195/taxreview.2014.32

Biggs, J. (2015). Citibank Is Working On Its Own Digital Currency, Citicoin. archive (consulté le 20 novembre 2015).

Borgia, F. (2005). Corporate governance \& transparency role of disclosure: how prevent new financial scandals and crimes. American University Transnational Crime and Corruption Center School of International Service.

De Filippi, P. (2016). The interplay between decentralization and privacy: the case of blockchain technologies. Journal of Peer Production, Issue, (7).

Humphrey, W. S. (1999). Bugs or Defects?. CMU SEI, 1.

Mitchell, S. L. (2007). GRC360: A framework to help organisations drive principled performance. International Journal of Disclosure and Governance, 4(4), 279296.https://doi.org/10.1057/palgrave.jdg.2050066 
Racz, N., Panitz, J., Amberg, M., Weippl, E., \& Seufert, A. (2010). Governance, risk \& compliance (GRC) status quo and software use: results from a survey among large enterprises.

Racz, N., Weippl, E., \& Seufert, A. (2011). Governance, risk \& compliance (GRC) softwarean exploratory study of software vendor and market research perspectives. In 2011 44th Hawaii International Conference on System Sciences (pp. 1-10). IEEE.https://doi.org/10.1109/HICSS.2011.215

Zheng, Z., Xie, S., Dai, H. N., Chen, X., \& Wang, H. (2018). Blockchain challenges and opportunities: A survey. International Journal of Web and Grid Services, 14(4), 352375.https://doi.org/10.1504/IJWGS.2018.095647 